\title{
Aspects culturels dans le manuel du français langue étrangère : étude de cas
}

\author{
Ariessa RACMADHANY \\ Département de français, Universitas Pendidikan Indonesia - Indonésie
}

Reçu le 25 octobre 2019 | Accepté le 31 décembre 2019

RÉSUMÉ. Un manuel reste toujours être considéré comme un principal matériel pédagogique pour la diffusion du savoir culturel français. Cette étude tente donc d'étudier l'aspect culturel dans le manuel du FLE. L'étude est menée en utilisant la méthode descriptive qualitative. Les objets de cette étude sont des textes tirés d'un manuel du FLE qui sont dispersées dans chaque chapitre avec des thèmes différents. Les données sont collectés en classifiant cinq dimensions de la culture comme les produits, les pratiques, les perspectifs, la communauté, et les personnes puis sont analysées en utilisant la théorie de Moran (2001). Les résultats montrent que ce manuel ont pu remplir les catégories des aspects culturels pour ses apprenants. Cette étude devrait donc contribuer au développement des recherches sur l'évaluation d'un manuel.

Mots-clés : analyse culturelle, culture, FLE, manuel

\begin{abstract}
A textbook is still considered as main educational material for the dissemination of French cultural knowledge. This study therefore attempts to study the cultural aspect in the French for Foreign Language (FFL) textbook. The study is conducted using the qualitative descriptive method. The objects of this study are texts taken from a FFL textbook which are scattered in each chapter with different themes. The data are collected by classifying five dimensions of culture such as products, practices, perspectives, community, and people and are then analyzed using Moran's framework theory (2001). The results show that this manual was able to fill the categories of cultural aspects for its learners. This study should therefore contribute to the development of research on the textbooks' evaluation.
\end{abstract}

Keywords: cultural analysis, culture, FFL, textbook

圈 auteur correspondant : ariessa.racmadhany@upi.edu

Pour citer cet article (Style APA) : Racmadhany, A. (2019). Aspects culturels dans le manuel du français langue étrangère : étude de cas. Francisola: Revue Indonésienne de la langue et la littérature françaises, 4(2), 150-160. doi: 10.17509/francisola.v4i2.24206

\section{INTRODUCTION}

La langue est l'un des nombreux éléments culturels universels, en plus des systèmes religieux, des organisations et systèmes sociaux, des systèmes de connaissances, des arts et autres (Koentjaraningrat, 1989). Dans son article, Purwoko (2010, p. 64) affirmait que les observateurs sociolinguistiques estimaient que la nature vivante ne pouvait être séparée de la vie socioculturelle de ses locuteurs. Les langues encore utilisées aujourd'hui doivent refléter les valeurs socioculturelles de sa zone géographique de pratique.

En 2016, l'Indonésie est entrée dans une ère de marché libre qui "oblige » indirectement les personnes à se mesurer les unes aux autres pour maîtriser les langues étrangères. Le français est une langue étrangère très attrayante pour les Indonésiens, cela se voit par l'existence de cinq Institut Français d'Indonésie et de deux 
Alliance Française sous la supervision de l'ambassade de France en Indonésie. Le français est par ailleurs la cinquième langue étrangère la plus parlée au monde avec 274 millions de locuteurs (Organisation internationale de la francophonie [OIF], 2014).

D'après Purwoko (2011) pour maîtriser une langue, il y a deux processus différents; le premier est la maîtrise de la langue maternelle, qui est un processus d'acquisition grâce à l'environnement socioculturel du sujet. C'est un phénomène automatique, commençant dès le plus jeune âge. Le second est quant à lui, un processus d'apprentissage, qui a pour but de maîtriser une langue étrangère. Dans son processus, un apprentissage nécessite un support pédagogique pour aider à comprendre cette langue étrangère. En général, les supports d'apprentissage utilisés sont les méthode scolaires.

La méthode est l'une des sources de supports pédagogiques pouvant aider les apprenants et les enseignants à atteindre les objectifs d'apprentissage. L'apprentissage est l'une des actions humaines effectuées en permanence afin de développer l'intelligence grâce à la capacité et la personnalité (Wulandari \& Saragih, 2018). Pour optimiser l'apprentissage, il faut être capable de développer des ressources qui touchent aux domaines cognitifs, affectifs et psychomoteurs. Parmi ces trois domaines, le domaine principal réside dans le domaine cognitif qui provient du cerveau humain en tant qu'organe important contrôlant les autres organes du corps. Le domaine cognitif peut être testé à travers la théorie d'apprentissage taxonomique ou la taxonomie d'apprentissage de Bloom.

Dans ce contexte, la définition fait référence aux théories de l'apprentissage de behaviorisme et de cognitivisme, où le behaviorisme considère que le " comportement » est une manifestation d'un processus d'apprentissage. $\mathrm{Qu}^{\prime}$ une personne réussisse le processus d'apprentissage ou non, cela se verra sur son comportement (Jamaludin \& Khoerudin, 2015, p.11). Lorsque le cerveau humain fonctionne, il affiche automatiquement le comportement résultant des processus cognitifs. Les processus cognitifs sont produits par l'interaction des apprenants avec le contenu de la méthode et avec l'intention d'exprimer le but d'un apprentissage. La relation entre les apprenants, la méthode et la théorie $\mathrm{d}$ 'apprentissage peut peuvent être illustrées dans le tableau suivant:

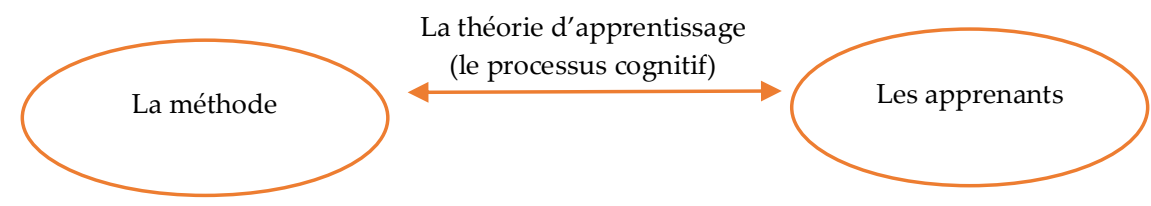

Figure 1. Le processus cognitif (Wulandari \& Saragih, 2018, p.25)

Soelaiman (1979, p.269) a constaté que la méthode est importante pour le développement de l'efficacité de l'apprentissage, en fonction de la manière de choisir et d'utiliser celle-ci. La sélection d'une méthode scolaire tiendra généralement compte de la possibilité pour les apprenants $\mathrm{d}$ 'apprendre par eux-mêmes sans les conseils constants de l'enseignant. Ainsi que les nombreux exercices pour les apprenants dans la méthode. Grâce à la méthode utilisée dans le processus d'apprentissage, les apprenants sont certainement non seulement en mesure de bien comprendre la matière, mais aussi de faire des exercices qui peuvent améliorer les connaissances de la matière qu'ils reçoivent en classe. Par conséquent, une méthode doit avoir des qualités pouvant répondre aux besoins d'un apprentissage avec certains critères.

Průcha (cité dans Tandlichová, 2003) considère que la méthode est un élément indissociable $\mathrm{du}$ processus enseignementapprentissage, car elle est un outil permettant 
aux enseignants de gérer leurs classes et, en même temps, un moyen d'apprendre de manière autonome. C'est donc la tâche des enseignants de rechercher, de choisir ou de modifier les matériaux pédagogiques qui seront remis aux apprenants (Bento, 2015).

Dans un manuel de langue, le contenu n'est pas seulement les points grammaticaux ou lexicaux mais aussi les points culturels sont l'un des critères de bonnes manuel de langue (Tagliante (1994). Des recherches précédentes (Wulandari \& Saragih, 2018; Shofiana, 2016; Kim \& Paek, 2015; Han \& Bae, 2005) ont aussi prouvé que le contenu culturel est une partie très importante pour l'apprentissage d'une langue.

Cependant d'après Kim \& Paek (2015), l'importance du contenu culturel dans les manuels a rarement été abordée dans les études antérieures, bien que la représentation des matériels liés à la culture ait un impact sérieux sur la manière dont les apprenants perçoivent les autres cultures. De plus, la plupart de ces recherches se sont concentrées sur l'anglais, le japonais ou l'arabe. Reconnaissant l'influence significative des manuels en langue étrangère sur la conscience culturelle et les connaissances interculturelles des apprenants, cette étude examine comment un manuel représente les cultures étrangères dans une perspective multiculturelle.

En plus la recherche de la contenu culturel pour les manuels du Français Langue Etrangère n'existe pas beaucoup. Pourtant la culture, est une chose étroitement liée à notre vie quotidienne. Mesthrie, Swann, Deumer et Leap (cité dans Sudartini, 2012, p. 47) définissent la culture comme « le mode de vie de ses membres; la collection d'idées et d'habitudes qu'ils apprennent, partagent et envoient de génération en génération ». La culture en ce sens est le projet de vie, qui détermine les modes et les formes de comportements appropriés ou acceptables dans certaines sociétés.

La culture est aussi un terme familier à notre vie quotidienne, mais en fait c'est un concept compliqué embrassant presque tous les aspects de la vie sociale humaine (Han \&
Bae, 2005). Aux fins de l'apprentissage des langues, Hammerly (1982) a identifié trois types de culture: la culture de l'information, la culture de la réussite et la culture du comportement.

La relation entre la culture et la langue joue un rôle important dans l'apprentissage des langues, car des arguments ont été avancés par des théoriciens tels que Valdes (1986), Byram (1989), Byram, Morgan et Colleagues (1994), Seelye (1993), qui mettent l'accent sur la valeur enseignement culturel pour acquérir des compétences linguistiques.

Après l'introduction de la théorie de la compétence linguistique de Chomsky, Hymes (1971) plaide sur l'importance de la faisabilité sociale des formes linguistiques, ce qui a contribué à la prise de conscience par les professeurs de langues que la communication implique des méthodes grammaticales grammaticalement correctes et culturellement acceptables dans l'utilisation du langage.

De nos jours, il ne fait aucun doute que l'enseignement culturel est devenu une partie acceptée de la pédagogie des langues et il est reconnu que la langue et la culture sont interdépendantes, qu'il n'est pas possible d'enseigner la langue sans culture et que la culture est le contexte requis pour l'utilisation de la langue (Han \& Bae, 2005).

C'est pourquoi ce sujet est un thème intéressant à aborder. En utilisant les perspectives de la dimension culturelle de Moran (2001) comme les produits, les pratiques, les perspectives, les communautés et les gens, nous avons aussi choisi un manuel principal du FLE en Indonésie.

$\mathrm{Au}$ cours du développement de l'apprentissage de français, de nombreux manuels de français ont été utilisés en Indonésie à la fois au lycée et à l'université. Telles que Campus, Taxi, Nouveau Rond-Point, Alter Ego, Version originale et l'une des plus récentes est Tendances.

Conformément aux arguments précédents, cette étude explore le type de culture présenté dans un manuel du FLE, à 
savoir Tendances pour le niveau A1. Par conséquent, les questions de recherche posées sont comme suit:

1. Comment ce manuel décrit les aspects culturel?

2. Dans quelle mesure ce manuel soutient l'interaction entre les cinq dimensions culturelles: produits, pratiques, perspectives, communautés et gens?

\section{MÉTHODE}

\subsection{Source de données}

Dans cet article, le chercheur a choisi d'analyser un manuel de français, à savoir Tendances A1 - Livre de l'élève. Cette méthode de français est l'une des dernières méthodes de français publiées par CLE International en 2016. Rédigé par Jacky Girardet, Jacques Pécheur, Colette Gibbe et Marie-Louise Parizet, ce manuel est destiné aux apprenants de langue française au niveau primaire, le niveau A1.

En Indonésie, le manuel Tendances A1 est également utilisée non seulement par des département de français dans les universités mais également au réseau de l'Institut Français d'Indonésie et de l'Alliance Française. Tendances A1 - Livre de l'élève est le nouveau manuel de français, il n'y a donc pas d'informations supplémentaires sur ce manuel. Par conséquent, nous avons besoin d'analyser de façon plus approfondie cette méthode afin qu'elle puisse être facilement utilisée par les enseignants et les apprenants de la langue française.

\subsection{Collecte de données}

Cette recherche utilise la méthode qualitative descriptive en prenant des textes dans la section « Point Infos » et en créant un corpus à partir de ces données. Il y a 18 textes faisant partie cette section.

\subsection{Analyse de données}

Pour analyser les données, le chercheur a utilisé la théorie de Moran (2001) qui est en effet le développement de la théorie de Sterns (1993). Sterns (1993) propose six sujets qui couvrent la plupart des aspects de l'enseignement de la culture que l'apprenant de langue typique semble avoir besoin. dans le but de maintenir les domaines d'un programme culturel à des proportions gérables, entre autres:

- les lieux (les emplacements physiques de la langue cible dans un ordre de priorité par les locuteurs natifs; les plans des rues ou les cartes)

- les personnes individuelles et les modes de vie (les styles de vie locaux, les idées, les valeurs individuels)

- les personnes et la société en général (les groupes divers par la stratification sociale)

- l'histoire (l'évolution historique; les perceptions historiques $\mathrm{du}$ locuteur natif)

- les institutions (le gouvernement, les écoles, la protection sociale, les institutions économiques, militaires et policiers, les institutions religieuses, les partis politiques, les médias)

- l'art, la musique, la littérature et d'autres réalisations majeures (les artefacts couramment acceptés dans la société cible).

Moran a ajouté un avis qui n'est pas très différent de l'avis de Sterns. Moran (2001) a soutenu que le concept de culture dichotomique était incomplet et a ajouté la «communauté» et les «personnes» comme autres facteurs pris en compte dans la dimension culturelle. Après avoir établi les cinq dimensions culturelles comme les produits, les pratiques, les perspectives, les communautés et les personnes, il a donné une définition de la culture. Selon lui, la culture est considérée comme un mode de vie qui se développe à partir d'un groupe de personnes et il s'agit d'une pratique partagée liée aux produits et aux perspectives et organisée dans un contexte social particulier (Moran, 2001).

Cinq dimensions ont été adoptées dans cette étude pour analyser comment les 
éléments culturels se reflètent et comment les cultures étrangères sont représentées dans chaque chapitre du livre Tendances A1. Le tableau 1 ci-dessous montre le résumé de cinq dimensions culturelle de Moran (2001).

Tableau 1. Résumé de cinq dimensions culturelles

\begin{tabular}{lll}
\hline Dimensions & Exemples & \\
\hline Produits & Artefacts & : nourriture, documents, langue, argent, outils \\
& $\begin{array}{l}\text { Lieux } \\
\text { Institutions } \\
\text { Fortiments, villes, maisons }\end{array}$ \\
& Fomille, droit, économie, religion, éducation, politique \\
& Opérations & : manique, vêtements, danse, peinture, film, architecture \\
Pratiques & Actes & : pratiques communicatives ritualisées \\
& Scénarios & : pratiques communicatives étendues \\
& Vies &
\end{tabular}

Perspectifs Ils représentent les perceptions, les croyances, les valeurs et les attitudes qui soustendent les produits et guident le comportement des gens dans la pratique de la culture. Ils peuvent être explicites mais souvent implicites, en dehors de la conscience consciente.

Communautés Ils incluent les contextes sociaux spécifiques (par exemple, les cultures nationales), les circonstances (p. ex. les cérémonies religieuses) et les groupes (p. ex. les différents clubs sociaux) dans lesquels les membres réalisent des les pratiques.

Personnes Ils se réfèrent à des membres individuels qui incarnent la culture et ses communautés de manière unique. L'identité personnelle et l'histoire de vie jouent un rôle clé dans le développement d'une personne culturelle.

\section{RÉSULTATS ET DISCUSSION}

3.1. Organisation de la rubrique Point Infos

Cette rubrique est un court texte contenant environ 30 à 100 mots de cette méthode contenu dans chaque chapitre de leçon. Plus le nombre de leçons abordées est élevé, plus le nombre de mots augmentent. Cette méthode contient neuf chapitres possédant plusieurs thèmes et étant complétés par environ deux " Point Infos». Ce qui suit est le titre des Point Infos de chaque chapitre.

Tableau 2. La rubrique Point Infos

\begin{tabular}{lll}
\hline Unité & Page & Les Titres de Point Infos \\
\hline Unité 1 & page 27 & Les Étrangers et Paris \\
& page 29 & Les Français et Les Réseaux Sociaux \\
Unité 2 & page 41 & Fêtes et Célébrations en France \\
Unité 3 & page 51 & Les Horaires en France \\
& page 55 & Le Dimanche en France \\
& page 57 & Le Nom de Famille \\
Unité 4 & page 62 & Les Sorties des Jeunes \\
& page 69 & Déjeuner « Vite Fait » \\
Unité 5 & page 79 & La SNCF \\
& page 83 & La France Touristiques \\
Unité 6 & page 93 & Payer en France \\
& page 99 & Petits Cadeaux entre Amis et Collègues de Travail \\
Unité 7 & page 105 & Les Sujets de Conversation \\
& page 111 & Les Veux du Jour de l'An \\
Unité 8 & page 121 & Les Religions en France \\
& page 125 & La chanson Francophone
\end{tabular}




\section{Unité 9 page 133 Les Français et Le Rêve du Départ à l'Étranger \\ page $141 \quad$ Où Habitent les Français?}

$\mathrm{Au}$ vu de chaque titre, nous nous apercevons que chaque « Point Infos » contient une information liée à la vie quotidienne des français. En outre, ces textes sont accompagnés d'illustrations sous forme de photographies qui aident les apprenants à mieux comprendre le contenu de ces textes.

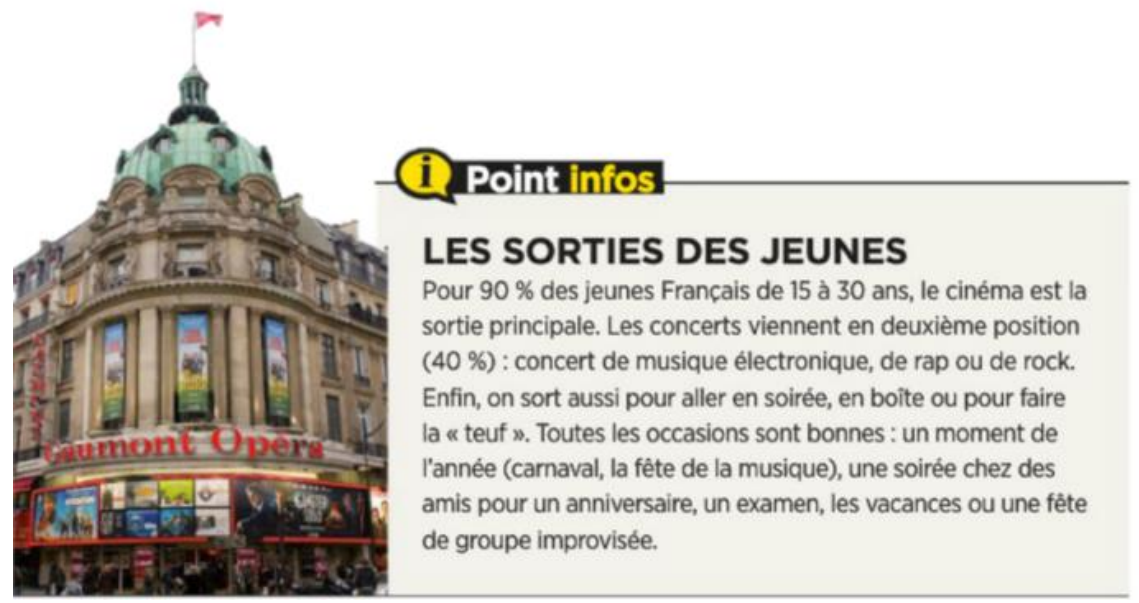

Figure 2. Texte de Point Infos - Unité 4 page 62

Voici une explication du contenu des textes de la rubrique Point Infos du méthode Tendances A1 - Livre de l'élève:

1. Les Étrangers et Paris, ce texte décrit le nombre d'étrangers et leur pays d'origine à Paris, capitale de la France. Il nous indique également quelques noms d'étrangers célèbres à Paris appartenant à divers domaines professionnels.

2. Les Français et les Réseaux Sociaux, dans ce texte, il y est expliqué quels sont les médias les plus souvent utilisés en France ainsi que leur pourcentage.

3. Fêtes et Célébrations en France: les noms des fêtes célébrées en France sont expliqués, y compris plusieurs fêtes religieuses différentes de Noël.

4. Les Horaires en France, ce texte explique les horaires de travail, les horaires à l'école et les horaires des magasins en France.

5. Le Dimanche en France, ce texte, explique que les dimanches en France, tous les magasins, à l'exception de ceux de Paris, sont fermés car le dimanche est un jour familial.

6. Le Nom de Famille, ce texte, nous parle des noms de famille couramment utilisés en France et comment ces nom sont utilisés chez un nouveau-né.

7. Les Sorties des Jeunes, ce texte explique les habitudes des jeunes en France ainsi que les lieux les plus visités lors de sorties entre amis.

8. Déjeuner «Vite Fait», ce texte explique les habitudes changeantes de la société française, en particulier le déjeuner, où les Français déjeunent vite même si la pause repas est longue.

9. La SNCF, ce texte présente la compagnie de chemin de fer française qui héberge différents types de trains, y compris le TGV (Train à Grande Vitesse) ou ce que nous connaissons comme « train rapide».

10. La France Touristique, explique le tourisme en France et quelles régions sont fréquemment visitées par les touristes étrangers.

11. Payer en France, ce texte explique le processus de paiement souvent utilisé 
en France, soit en espèces, soit par chèque.

12. Petits Cadeaux entre Amis et Collègues de Travail, ce texte décrit le moment où les Français donnent généralement des cadeaux à leurs amis.

13. Sujets de conversation, explique ce qui est habituellement discuté et ce qui ne devrait pas être discuté avec les Français.

14. Les Vœux du Jour de l'An, est à propos $\mathrm{du}$ changement d'habitude pour féliciter quelqu'un ou célébrer un évènement spécial. $C$ 'était initialement l'envoi d'une carte de vœux mais maintenant, c'est plutôt un envoi de message par voie électronique.

15. Les Religions en France, ce texte décrit quelle religion est suivie en France, ainsi que le pourcentage d'adhérents et ceux qui pratiquent réellement les enseignements de leur religion.

16. La chanson francophone, explique le développement des chansons francophones, les chansons produites par des francophones qui ne sont pas citoyens français.

17. Les Français et le rêve du départ à l'étranger explique le nombre de jeunes français qui poursuivent leurs études à l'étranger.

18. Où Habitent les Français?, présente la résidence idéale pour les Français.

\subsection{Aspects culturels de Point Infos}

En outre, lors de la discussion précédente, les cinq dimensions culturelles ont été présentées et seront abordées à partir de la rubrique «Point Infos ». Ces questions sont des points qui devraient être pris en compte par les compilateurs de manuel, en particulier les manuels de langues étrangères.

Basé sur les critères de Moran (2001), voici les analyses de cinq dimensions culturelles dans la rubrique « Point Infos ».

\subsubsection{Produits}

Le «produit», d'après Moran (2001), est défini comme un système de codes qui mesure les dimensions culturelles sous quatre grands aspects: les artefacts, les lieux, les institutions et les formes d'art. Les artefacts incluent des questions culturelles telles que la nourriture, la langue et l'argent, tandis que les lieux font référence à des bâtiments ou des villes liés à une culture particulière. Les institutions mesurent des sujets culturels qui sont combinés avec le droit, la famille et la politique, et les formes d'art incluent des sujets culturels liés à la musique, la danse ou la peinture.

Dans ce manuel, la représentation du «produit» montre dans quelques «Point Infos » par exemple dans le texte « La France Touristiques » montrant les lieux touristiques en France.

La France reçoit chaque année 85 millions de visiteurs. On connaît ses grandes destinations touristiques: Paris et ses monuments, la Normandie et son abbaye $d u$ Mont-Saint-Michel, les châteaux de la Loire, la côte d'Azur, le Sud, ses paysages et le pont $d u$ Gard...et aussi les parcs d'attraction (Disneyland, le parc Astérix, le Futuroscope). Mais le reste du pays a aussi son intérêt et la France est un pays très varié. Chaque région a ses paysages, son histoire et ses traditions. (Pécheur et al, 2016, p. 83)

Le texte « Où habitent les français ?» montre aussi le même aspect, les lieux où les Français habitent.

Quand on interroge les Français sur leur logement idéal, $72 \%$ souhaitent une maison individuelle avec jardin et piscine, située à la campagne. Ces souhaits sont en partie satisfaits : 57\% des Français vivent dans une maison contre $43 \%$ dans un appartement. Mais seulement $18 \%$ de ces maisons sont à la campagne. Les autres sont situées dans les villes ou leurs agglomérations (une agglomération comprend une ville, sa banlieue et des villages réunis à la ville). (Pécheur et al, 2016, p. 141)

Puis, le contenu sur « l'artefact » est montré dans le texte «Payer en France». Ce texte parle de la monnaie utilisée en France et comment les Français payent des achats.

L'euro est la monnaie de la France et de 18 pays d'Europe. Pour un achat de moins 10 
euros, on paie souvent en espèces (en liquide). Au-dessus, on paie par carte bancaire ou par chèque. Les commerçants n'aiment pas beaucoup les gros billets. En France, on voit très peu de billets de 100 ou de 500 euros. Dans beaucoup de pays d'Europe comme l'Allemagne ou le Danemark, on n'utilise plus le chèque. En France, $14 \%$ des achats sont payés par chèque. (Pécheur et al, 2016, p. 93)

Et pour montrer le terme de $\mathrm{l}^{\prime}$ " institution ", il existe un texte sur "Le nom de la famille». Ce terme parle de la famille et le texte montre la construction du nom de famille en famille française.

Un enfant peut avoir comme nom de famille: le nom de son père ( $83 \%$ des cas): Paul Durand; le nom de sa mère: Paul Lambert. Il peut aussi utiliser les deux noms: Paul Durand-Lambert / Paul Lambert-Durand. Quand une femme se marie elle garde son nom de famille (Marie Vernet). Ele peut aussi utiliser le nom de son mari (Marie Durand) ou les deux noms (Marie Vernet-Durand ou Marie Durand-Vernet). Un homme marié peut aussi prendre le nom de famille de sa mère (cas très rare). (Pécheur et al, 2016, p. 57)

\subsubsection{Pratiques}

La «pratique» caractérise d'autres dimensions culturelles qui mesurent des divers aspects de la pratique culturelle en termes d '«opérations», d'«actions», de «scénarios» et de «vie»(Moran, 2001). Plus précisément, «opérations » se réfèrent à la pratique de la manipulation des produits culturels. Tandis que les «actions" soient une pratique de communication rituelle et les «scénarios » se réfèrent à des pratiques de communication étendues. Et le dernier point, c'est la «vie » dont le contenu est une description ou une histoire de membres de cette culture.

La représentation des «pratiques» est trouvée dans les textes suivants : Les horaires en France, Le dimanche en France, Les sorties des jeunes, Petits cadeaux entre amis et collègues de travail, et Les vœux de jour de l'An. «Les horaires en France» montre la situation de vie des Français et aussi leurs habitudes de tous les jours en montrant les heures.
Généralement, les Français déjeunent entre $12 \mathrm{~h}$ et $13 \mathrm{~h} 30$. Il dînent entre $19 \mathrm{~h}$ et $20 \mathrm{~h} 30$. Les magasins sont souvent ouverts de $10 \mathrm{~h}$ à $19 \mathrm{~h}$ du lundi au samedi. Les banques sont ouvertes de $9 h$ à $17 h$ du lundi au vendredi. Les enfants vont à l'école le lundi, le mardi, le jeudi, le vendredi de 8 h30 à 11 h30 et de $13 h 30$ à $16 h 30$ et le mercredi matin. (Pécheur et al, 2016, p. 51)

Le texte « Les sorties des jeunes » montre les pratiques que les jeunes français font pour leur loisir.

Pour 90\% des jeunes Français de 15 à 30 ans, le cinéma est la sortie principale. Les concerts viennent en deuxième position $(40 \%)$ : concert de musique électronique, de rap ou de rock. Enfin, on sort aussi pour aller en soirée, en boîte ou pour faire la " teuf ». Toutes les occasions sont bonnes : un moment de l'année (carnaval, la fête de la musique), une soirée chez des amis pour un anniversaire, un examen, les vacances ou une fête de groupe improvisée. (Pécheur et al, 2016, p. 62)

L'aspect «pratique» qui présente dans le texte « Petits cadeaux entre amis et collègues de travail », est l'habitude des français quand il $\mathrm{y}$ a une fête ou le moment ils doivent apporter des cadeaux ou des choses.

En France, on offre un cadeau à ses amis :pour leur mariage (il y a souvent une liste de cadeaux souhaités par le couple), à leur anniversaire ou pour les fêtes de fin d'année ; quand on est invité chez eux. On peut alors apporter des fleurs, une bouteille, un livre ou un cadeau original. On peut aussi décider avec ses amis d'apporter le dessert ou un plat ; quand on revient d'un long voyage. À un collègue de travail, on peut offrir un cadeau (souvent collectif) à l'occasion de son départ : départ à la retraite, départ pour une autre entreprise ou un autre service. Quand on reçoit un cadeau, on l'ouvre et on dit quelques mots de remerciement. (Pécheur et al, 2016, p. 99)

Aussi pour le texte «Les vœux du jour de l'An » où il y a des traditions que les Français font.

Les væux du jour de l'An sont une tradition. $C^{\prime} e s t$ l'occasion de rester en contact avec les membres de sa famille ou avec ses amis et des connaissances. On envoie de moins en moins de cartes par la poste mais plutôt par internet. Ou bien, on fait un courriel personnalisé. On peut présenter ses vœux un peu avant Noël : "Je vous souhaite de 
joyeuses fêtes et une heureuse année... ». Mais on le fait plutôt dans les premiers jours de janvier: "Je vous souhaite une heureuse année ... une bonne santé ... beaucoup de succès ... ». (Pécheur et al, 2016, p. 111)

\subsubsection{Perspectifs}

Les «perspectifs», d'après Moran (2001), incluent le matériel culturel qui transmettent certaines perceptions, valeurs et croyances et qui guident le comportement des gens dans les pratiques culturelles. Par exemple, le texte «Les sujets de conversation» décrit dans ce manuel peut être classé dans la catégorie «perspectif», car il reconnaît les perceptions des Français sur quelques questions considérées taboux pendant une conversation. Il y a aussi un texte sur le croyance titré « Les réligion en France » et un texte sur la valeur ou la perception, par exemple «Les Français et le rêve du départ à l'étranger.»

« Les religions en France » montre que la France est un pays laique car les religions considèrent comme une affaire privée.

La France est un pays où les religions sont une affaire privée. $60 \%$ des Français sont catholoques mais très peu pratiquent leur religion (5\%). Beaucoup de ces pratiquants ont plus de 65 ans. $7 \%$ sont musulmans (30\% de pratiquants). $4 \%$ sont protestants ( $40 \%$ de pratiquants). $1 \%$ est de religion juive. $2 \%$ pratiquent une autre religion (bouddhisme, etc.). $26 \%$ se disent sans religion. (Pécheur et al, 2016, p. 121)

Et dans le texte «Les Français et le rêve du départ à l'étranger ", les auteurs montrent la préférence des jeunes français pour leur travail et leurs études.

Comme un peu partout dans le monde des jeunes Français voyagent à l'étranger et beaucoup rêvent d'aller $y$ vivre. On dit aussi : "Les jeunes très qualifiés ne restent pas en France. Ils préferent partir à l'étranger. » La réalité est différente. Il y a seulement 1,6 millions de Français qui sont installés à l'étranger (2,4\% de la population). Le nombre de diplômés (ingénieurs, scientifiques, etc.) qui partent est stable (entre 10 et $15 \%$ depuis 10 ans). Il y a beacoup plus d'étudiants étrangers en France (300.000) que d'étudiants français à l'étranger (70.000). (Pécheur et al, 2016, p. 133)

\subsubsection{Communautés}

Les «communautés» décrivent le contexte social, les circonstances et les groupes particuliers dans lesquels les membres s'engagent dans des pratiques culturelles (Moran, 2001). Les cérémonies religieuses et les clubs sociaux et culturels sont des exemples de contenu qui seront inclus dans la catégorie de " communauté». Le texte trouvé sur ces sujets sont «Les Français et les réseaux sociaux »

$85 \%$ des Français utilisent internet. Les réseaux sociaux populaires sont Facebook (63\%), Skype (47\%), Copains d'Avant (32\%), Google+ (32\%), Deezer (28\%), Youtuber (27\%), Twitter (17\%) et LinkedIn (14\%). (Pécheur et al, 2016, p. 29)

Et dans le texte «Fêtes et célébrations en France », des lecteurs peuvent connaître qu'en France il existe les communauté réligieuses et aussi culturelles.

Les Français fêtent Noël (en famille), le jour de l'An (avec des amis ou en famille), le 14 juillet (fête nationale), la Toussaint (1er novembre, jour des morts). Il y a aussi : les fêtes locales (la féria de Nìmes, le carnaval de Dunkerque...), les fêtes religieuses (l'Aïd pour les musulmans, Noël pour les chrétiens, Roch Hachana pour les juifs), les fêtes des communautés (le Nouvel An chinois). (Pécheur et al, 2016, p. 41)

\subsubsection{Personnes}

La dimension des «personnes» font référence à des membres individuels qui représentent une certaine culture et communauté (Moran, 2001). Deux textes, La chanson francophone et Les étrangers à Paris, sur les personnes populaires en France ,même s'ils ne sont pas français, décrits dans ce manuel peuvent être un bon exemple des aspects culturels des «personnes».

Le texte "La chanson francophone» représente les étrangers qui chantent en français et deviennent très populaire en France.

Quand on parle de "chanson française ", on devrait dire "chanson francophone». Beaucoup de musiciens ou de chanteurs qui sont devenus célèbres en France sont originaires d'autres pays où on parle français. Aujourd'hui, Stromae vient de Belgique comme Jacques Brel dans les années 
1960. Ils ont apporté des styles nouveaux qui ont enrichi la chanson française traditionnelle: la country et la folk canadienne avec le Québécois Roch Voisine ou la Québécoise Cour de pirate, le raï algérien avec Faudel, le reggae avec Alpha Blondy, le $R^{\prime} n^{\prime} B$ avec Corneille, un Rwandais qui vit au Canada, et bien d'autres. (Pécheur et al, 2016, p. 125)

Puis «Les étrangers à Paris » présent aussi les personnes populaires de beaucoup de pays étrangers qui font partie de la vie parisienne.

Il y a à Paris $12 \%$ d'étrangers. Ils viennent d'Afrique du Nord (Algérie, Maroc, Tunisie), des pays de l'Afrique francophone, d'Europe (Espagne, Italie, Portugal, etc.) ou d'Asie (Vietnam). Des étrangers célèbres à Paris :

- Des artistes: Blanca Li (choréographe espagnole), Marjane Satrapi (auteur de $B D$ et cinéaste iranienne), Miquel Barcelo (peintre espagnol), Andy Gardiner (DJ anglais), Arik Levy (designer israélien) ;

- Des écrivains: Zoé Valdés (cubaine), Nancy Huston (canadienne), Douglas Kennedy (américain), Yasmina Khadra (algérien);

- Des comédiens: Juliana Carneiro da Cunha (brésilienne) ;

- Des hommes politiques :Hô Chi Minh (vietnamien).

(Pécheur et al, 2016, p. 27)

\section{CONCLUSION}

En se basant sur la formulation des problèmes et le but de la recherche ainsi que sur les résultats de l'analyse, nous pouvons conclure que l'organisation de courts textes dans la rubrique «Point Infos » à l'aide de photographies supplémentaires est très utile pour aider les apprenants à comprendre le contenu du texte. En outre, le nombre de mots dans chaque texte, de 30 à 100 mots, facilite également la compréhension du texte par les apprenants, en particulier pour le niveau linguistique de base (A1), à l'aide des explications fournies par le professeur.

De plus, tous les textes «Point Infos» présentés dans ce manuel ont pu remplir les catégories des aspects culturels pour ses apprenants. Ils pourraient bien comprendre la culture montrée dans ce manuel à l'aide de leur professeur de français.
Dans le cadre de l'apprentissage du français à l'université, cette rubrique Point Infos peut être utilisée dans le cours de Compréhension Écrite 1 (au premier semestre) ou de Civilisation Française. L'équipe d'auteurs du manuel a compris que le contenu culturel est un aspect important de l'apprentissage d'une langue étrangère, en particulier pour la langue française, riche culturellement parlant.

Ainsi, en utilisant cinq dimensions de la culture, à savoir les produits, les pratiques, les perspectifs, les communautés, et les personnes observés dans cette étude, les lecteurs peuvent obtenir un indice dans le manuel Tendances A1 - Livre d'élève grâce à la rubrique Point Infos et son contenu culturel lié à la France.

\section{REMERCIEMENTS}

Nous tenons à remercier tous les organismes et/ou toutes les personnes qui ont apporté une contribution significative à la rédaction et/ou à l'amélioration de l'article.

\section{RÉFÉRENCES}

Bento, M. 2015. Manuels de français langue étrangère et pratiques d'enseignants. Recherches en didactique des langues et des cultures. Recherches en didactique des langues et des cultures, pp. 1-14. URL : http://rdlc.revues.org/739

Byram, M. 1989. Cultural studies in foreign language education. Clevedon, UK: Multilingual Matters.

Byram, M., Morgan, C., \& Colleagues. 1994. Teaching and learning language and culture. Clevedon, UK: Multilingual Matters.

Hammerly, H. 1982. Synthesis in second language teaching. Blain, Washington: Second Language Publications.

Han, Y. \& Bae, Y. 2005. An analysis of the cultural content of high school and college English textbooks. English Teaching 60(4), pp.47-70.

Hymes, D. 1971. Competence and performance in linguistic theory. In R. Huxley \& E. Ingram (Eds.), Language acquisition: Models and methods, pp. 328. London: Academic Press. 
Jamaludin, Komarudin, A., \& Khoerudin, K. 2015. Pembelajaran Perspektif Islam. Bandung: PT. Remaja Rosdakarya.

Kim, S \& Paek, J. 2015. An analysis of culture-related content in English textbooks. Linguistic Research 32 Special Edition, pp.83-104.

Koentjaraningrat. 1989. Pengantar Ilmu Antropologi. Indonesia : Aksara Baru.

Lestari, L. 2010. Mengenalkan Aspek Budaya dalam Pembelajaran Bahasa Inggris. Lingua Didaktika Volume 3 (2), 145-151.

Moran, P. \& Ziwen, L. 2001. Teaching culture: Perspectives in practice. Boston, MA: Heinle \& Heinle.

Organisation Internationale de la Francophonie (OIF). (2014). 274 millions de francophones dans le monde. (cité de https://www.francophonie.org/274millions-de-francophones-dans.html )

Pécheur, J., Girardet, J., Gibbe, C., \& Parizet, M. 2016. Tendances A1 - Livre d'élève. Italie : CLE International.

Purwoko, H. 2010. Muatan Sosial-Budaya dalam Buku Teks Pelajaran Bahasa Asing. Jurnal Parole, Vol. 1 (10), pp. 6477.

Seelye, H. 1993. Teaching culture: Strategies for intercultural communication. Lincolnwood: National Textbook Company.

Shofiana, L. 2016. Muatan Budaya dalam Buku Texte Pelajaran Bahasa Arab untuk Siswa Madrasah Aliyah Kelas X Kurikulum 2013 Terbitan Kementerian
Agama (Tinjauan Analisis Teori Michael Byram). Tesis Program Studi Pendidikan Islam Konsentrasi Pendidikan Bahasa Arab, Program Pascasarjana UIN Sunan Kalijaga, Yogyakarta: tidak diterbitkan.

Soelaiman, D. 1979. Teori dan Praktek Pengajaran. Semarang: IKIP Semarang Press.

Sterns, H. 1993. Issues and options in language teaching. Oxford: Oxford University Press.

Sudartini, S. 2012. Inserting Local Culture in English Language Teaching to Promote Character Education. Jurnal Pendidikan Karakter, Tahun II (1), pp.4554.

Syairi, K. A. 2013. Pembelajaran Bahasa dengan Pendekatan Budaya. Jurnal Dinamika Ilmu Vol. 13, pp.174-188.

Tagliante, C. 1994. La Classe de Langue. Paris : CLE International.

Tandlichová, E. 2003. EFL coursebook in learner-centred learning and teaching. In Theory and Practice in English Studies, 1, pp.145-151.

Valdes, J. M. (Ed.). 1986. Culture bound: Bridging the cultural gap in language teaching. Cambridge: Cambridge University Press.

Wulandari, R. \& Saragih, F. 2018. Analisis Buku Ajar Marugoto: Bahasa dan Kebudayaan Jepang A1 Ditinjau Dari Ranah Kognitif Taksonomi Bloom. Jurnal Pendidikan dan Pengajaran Bahasa Jepang, Vol.3 no.1, pp. 24-37. 